\title{
Liposuction Contouring After Head and Neck Free Flap Reconstruction
}

Amir E Ibrahim*, Hamed Janom, Mohamad Raad

Division of Plastic Surgery, Department of Surgery, American University of Beirut Medical Center, Lebanon

*Corresponding author: Amir E Ibrahim, Faculty Member, Division of Plastic Surgery, Department of Surgery, American University of Beirut Medical Center, Lebanon, Tel: +9613720594; E-mail: ai12@aub.edu.lb

Received date: May 28, 2014, Accepted date: April 22, 2015, Published date: April 27, 2015

Copyright: () 2015 Ibrahim AE et al. This is an open-access article distributed under the terms of the Creative Commons Attribution License, which permits unrestricted use, distribution, and reproduction in any medium, provided the original author and source are credited.

\begin{abstract}
Resection of bulky head and neck tumors is typically followed by microvascular free flap reconstruction. The latter has shown an acceptable success rate but often requires a secondary revision with a free tissue transfer reconstruction to improve outcome; both cosmetic and functional. Direct surgical revision via electrocautery/scalpel poses a high risk of flap perfusion compromise. Suction assisted lipectomy on the other hand is a feasible and safe technique that offers favorable contouring with comparable restoration cosmetic and functional outcomes. In this article, we review the indications and advantages of this technique and provide an outlook on its safety and pitfalls.
\end{abstract}

Keywords: Liposuction; Free Flap; Reconstruction

\section{Introduction}

Free tissue transfers are used in the correction of head and neck defects following tumor excision or burn debridement $[1,2]$. The surgical outcome can be affected by several parameters such as flap characteristics (thickness and bulk, adiposity, skin elasticity), flap contracture, postoperative radiotherapy, and muscle atrophy [2]. A wide variety of flaps have been employed in primary reconstruction, the ultimate cosmetic result is however still often unpredictable [3] requiring secondary intervention. One commonly employed approach consists of reconstructing the defect with a flap larger than necessary, because it will likely contract following radiation. That is followed by a secondary intervention in which the flap can be easily revised with liposuction and direct excision to achieve a more cosmetic contour or optimize speech and swallowing functions $[2,3]$.

Primary thinning of large myocutaneous and fasciocutaneous flaps at the time of free flap transfer, doing so risks damaging the blood supply to the skin, particularly in the vicinity of the cutaneous perforating blood vessels [4]. One possible alternative is primary thinning using microdissection around the perforators, but is tedious and requires fine techniques. Previously, secondary refinement constituted of peripheral or central incisions to elevate the flaps and direct excision of fat and scar tissue [5]. This technique may result in suboptimal outcomes due to limited and incomplete excision of flap areas around the vascular pedicle; furthermore, excisions around the perforators can cause partial or total flap loss along with potential wound-healing complications even with peripheral revascularization $[1-3,6]$.

Therefore, secondary contouring, by debulking and thinning of healed flaps, is usually performed at a time when the flap is no longer entirely dependent on perforating blood vessels $[3,7]$. The ability to thin the subcutaneous tissue of the entire flap allowed a greater degree of skin paddle advancement and therefore greater contour improvement than would have been realized using only direct excisional techniques [2]. Usually, and as reported by post-suction endoscopic evaluation, flap loss following conventional liposuction is theoretically minimal since fibrous structures containing blood vessels remains unharmed as the fat is removed with liposuction [8].

Suction assisted lipectomy (SAL) was first introduced by Hallock in 1985 [5,9] to debulk pedicled or microvascular free flaps followed by Baird and Nahai [10] in 1989, and later by Wooden et al. in 1993 who analyzed a series of 12 patients undergoing liposuction for flap debulking [2]. It has now been adopted for microvascular free flap revision [11] and has become a method widely resorted to since it offers microsurgeons the ability to thin the entries subcutaneous tissue of a flap with minimal risk of injury to the perforator blood supply or pedicle. This method offers the ability to thin the entire subcutaneous tissue without the potential risk to the perforator vessels as in the direct excision approach and hence a less risk of partial or full flap loss [1-3,5-7,9-17]. This technique is safe since it is known that flap revascularization does occur and comes from the wound bed itself. This allows subsequent manipulation of the blood supply without jeopardizing the flap that remains intact following liposuction $[8,10,18]$. Liposuction, scar revision and re-insetting the redundant flap skin can improve functional outcomes such as speech and swallow, oral competence, nasal breathing, visual field, or a better skin quality around a tracheotomy [19].

Furthermore, this method can be applied to other areas of nonfolded free flap debulking such as head and neck resurfacing, and upper extremity and lower extremity reconstruction [7]. In addition, it is recommended that only circumferential flap edges should be violated while removing excess skin to better maintain peripheral vasculature patency as much as possible [8]. Furthermore, even though more data from clinical trials remains to be elucidated, an ultrasonic surgical aspirator may have less traumatic effect and hence a potential safer method $[20,21]$.

\section{Technique}

A topographic map of the flap can be drawn using a Sterile Surgical Skin Marking Pen to predetermine the recontouring flap field. Lidocaine and epinephrine may be preinjected to help minimize bleeding. A small skin incision is made to allow the introduction of the suction cannula into the subcutaneous plane of the flap. The choice of cannula is discussed below. The incisions are done at positions that 
Page 2 of 5

will allow the cannula to reach the widest area of the subcutaneous plane of the flap. A blunt dissection with artery forceps can help the portal of entry for the suction cannula. The cannula is connected to a vacuum syringe and manual suction is performed. Compared to the use of a vacuum machine, this allows better control of the amount of fat removed, accurate contouring and safer on the flap. The fat is removed by rapid back and forth strikes with the suction cannula. The surgeon's non-dominant hand can be used to direct the cannula and to try to decrease bleeding by preserving $5 \mathrm{~mm}$ of subdermal fat as assessed by the thickness between the cannula and the external skin. It is also advised to avoid the flap margins to avoid any injury to microanastomosis or collateral vasculature. The time and number of strokes needed depends on the location, the size, and the fat content of the flap. Any excess tissue is later removed by excision and flap advancement. It is however recommended that only circumferential flap edges should be violated while removing excess skin to better maintain peripheral vasculature patency as much as possible [8]. Bulky compressive dressing may be used postoperatively to reduce seroma and hematoma.

\section{Technical Aspects}

There is no cannula proven to be superior for all circumstances. Several parameters can come into play in choosing the best cannula; the patient's overall health, areas of the body to be treated, the technique chosen and the volume of aspirate come into play when choosing the proper cannula. However, the usage of blunt tip cannulas as compared to a sharper and more pointed cannula may cause less damage to the surrounding area and hence may help preserve flap vascular patency [22]. Also to facilitate fat aspiration with less effort to avoid subsequent trauma, choosing a cannula with multiple opening requires less effort while suctioning [22]. Awareness of pedicle location is recommended and avoidance of injury by liposuction cannula can help maintain pedicle vasculature and nerves.

The use of dry, wet, super-wet, or tumescent techniques has not shown have different success rates. Hence, the decision to use a certain modality is purely dependent on the surgeon's decision and familiarity with that technique [22]. The surgeon's decision might depend on several factors including the patient's overall health, body mass index, the estimated volume of aspirate to be removed, and any concurrent surgical procedures.

Medium depth liposuction is preferred for a planned scar revision and skin excision when more one third of the flap periphery elevation is anticipated (Figure 1).

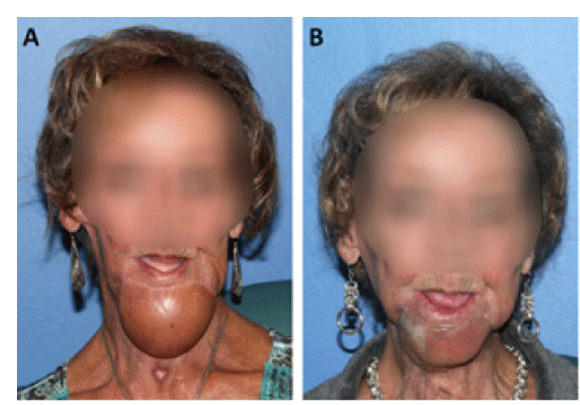

Figure 1: SAL in a patient with a mandibular and chin defect postradiation therapy.
65 year-old female who is six months after a double free flap reconstruction (Fibula osteo-cutaneous flap and Anterolateral thigh flap) for a radiated, through and through, composite mandibular and chin defect. Patient underwent intra and extra-oral medium depth SAL with direct redundant skin excision that improved her aesthetic and functional outcomes (A) Preoperative view, (B) Postoperative view (six weeks after SAL).

Superficial liposuction [14] is ideal for mild debulking and contouring without skin excision that provides a certain level of skin tightening (Figure 2). Figure 2 illustrates superficial liposuction for a moderately bulky anterolateral thigh free flap used for radiated, through and through, cheek defect six months from the microsurgical reconstruction.

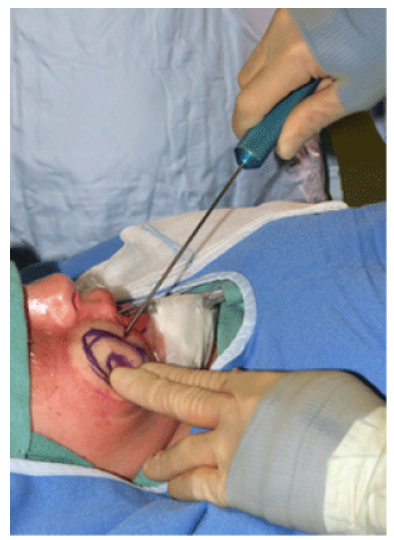

Figure 2: SAL Intraoperative picture.

\section{Timing of Liposuction}

Secondary interventions to any free flap transfer should be delayed for several months after the initial free flap transfer [1]. This allows more time for a complete neovascularization prior to secondary intervention by giving the tissue suffice time to recover from surgical edema and inflammation and any acute effects of radiation [1].

Historically, a 3 month delay before the secondary revision was considered for the edema to resolve, all wounds to heal, and the revascularization to take place to allow it to become pedicle independent $[2,6,10]$. Currently, most secondary reconstructions with flap debulking and revisions with SAL are done at least 3 to 6 months from the primary reconstruction with good surgical and patient satisfactory outcomes $[12,13,17,23-29]$. Many large series average at around nine months of waiting [6] even though a sign of ischemia to the tissue was observed at up to 15 months of delay [6] with no differences in observed outcomes at both delay times extremes. For example, in a series on 22 patients who had undergone reconstruction with free ATL flaps, Hung et al. used a non-standard open tip cannula for liposuction and w-plasty for scar revision in a one-stage operation in oral through-and-through defect cases. The average waiting time was 141 days after primary reconstruction. The authors report good aesthetic results with an $85 \%$ of patients reporting satisfaction with the outcome. Currently at the University of Texas MD Anderson Cancer Center, a three months delay is advised prior to any reconstruction to non-irradiated flaps. This however may not apply to all cases, as a 
Page 3 of 5

longer delay of 6 months is given to oncological cases with flaps undergoing post-operative radiation therapy as discussed later.

\section{Discussion}

\section{Debulking and contouring}

SAL improves head and neck flap contour by decreasing the adiposity of the flap [30]. SAL can be applied in the following conditions: Myocutaneous or fasciocutaneous flaps with mild bulkiness where no or minimal skin excess resection for scar revision is expected or with substantial bulkiness to be utilized in combination with direct excision of skin and fat [31], and also in the reconstruction of adipofascial flaps that are completely buried under the normal facial or neck skin $[31,32]$.

In the secondary revision, a proper thickness of the reconstructed site should be used to estimate graft thickness [32]. The anterolateral thigh (ALT) flap can be thinned through several techniques [32]. The adipofascial ALT flap has had a good success rate in repairing several types and sizes of soft tissue deficits as it has shown superior aesthetic and functional outcomes. In addition, it has also demonstrated lower morbidity at donor sites as compared to adipocutaneous flap [27,28]. The reason behind this is the ALT flap's solid blood supply that allows secondary intervention with a decreased percentage of vascular complications.

Furthermore, if primary thinning of the adipofascial ALT flap does not compromise the vasculature of the subfascial plexus, this gives the primary free flap transfer a higher survival rate. The decreased vasculature related complications can be explained by the anatomy of the perforating vessels that run perpendicular after passing the deep fascia $[27,28]$. The flap is also anchored by the deep fascia to the underlying structures preventing linkage or sagging as in the intraoral cavity [33]. Last, the relative uniform skin color of the ALT in facial correction avoids the patch-like appearance on the corrected facial area.

\section{Scar revisions and skin resurfacing}

Commonly, fasciocutaneous or myocutaneous free flaps are inset in a hostile field where the skin paddle is surrounded by irradiated skin excision to match skin thickness surrounding the recipient defect. When healed, flap bulkiness is exacerbated by the surrounding contracted irradiated and scarred skin can that can create a significant aesthetic deformity. Liposuction deflation of these flaps creates a reverse tissue expansion effect that produces excess amount of healthy and non-radiated skin that is commonly used to replace poor quality skin surrounding the flap [34]. If thinning is extreme, it is rare though possible to lose the whole flap due to an inadvertent damage to the main pedicle or perforator. Liposuction for primary debulking obviates the need for excessive thinning of free flaps and the risk of possible disastrous consequences.

\section{SAL in Irradiated flaps}

Radiation therapy (RT) has immediate and time-dependent effects on soft tissue. It has long been established that radiation therapy compromises blood supply by significantly explained by vessel fibrosis and fibrinoid necrosis [35], and a decreased healing phase explained by slower fibroblast proliferative phase [36]. Free flaps provide the advantage of having the vasculature transferred with the free flap itself.
This provides a better blood supply to both the donor tissue and recipient site and this improved functional has especially been noted in irradiated tissue with compromised blood supply as discussed earlier [37].

Although SAL is considered a safe and reliable option for flap reconstruction, surgeons have shied away from its use on irradiated flaps that may explain the paucity of literature on its efficacy in the reconstruction of irradiated flaps. The few available studies describing the efficacy and safety of SAL in non-irradiated flaps vs irradiated flaps conclude that a minimal difference in outcomes is observed between both groups $[1,31]$.

The delay before the secondary intervention for revision in radiated flaps did however have an impact on the results. This may be explained by the fact that RT has a severe immediate effect on soft tissue as well as a progressive time dependent arteriolar and capillary fibrosis. One study by Bui et al. describes satisfactory results and no significant differences when using SAL in irradiated vs non-irradiated flaps given a longer delay time is considered for irradiated flaps as described in their 41 patient series (6.9 vs 9.6 months respectively) [1]. Another study by Ellsworth et al. concluded no difference between both groups even when correcting for the delay time since complications had been seen in patients with a delay time at both extremes. In both studies, the RT dosages were between 60 to $64 \mathrm{~Gy}$ and the delay time was between 6 to 38 weeks after the last RT given $[1,31]$.

The results of both studies show that SAL achieves acceptable cosmetic results in irradiation skin, the time required before a secondary intervention whether after the flap transfer or the last RT remains controversial, but we still recommend a longer delay before secondary intervention. Larger studies are still needed to assess the optimal parameters with the best surgical outcome.

\section{Safety and Pitfalls}

\section{Factors predicting optimal results}

Data consensus on what patient factors best predict optimal results in SAL is yet to be elucidated. Most data available stresses on aiming for an initial flap transfer and construction success. Proper patient selection is also of paramount importance [38]. Several case series, including a 500 free-flap reconstructions series [39], have outlined the major risk factors including postoperative [40] and preoperative [41] radiation [42], neoadjuvant or postoperative chemotherapy [42], patient age [39,41], ASA class [41], smoking [39,41], diabetes [38] and salvage free flap transfer [38]. One study demonstrated no correlation of radiation, smoking, diabetes, or pulmonary disease [43].

\section{Oncological safety}

Oncological patients are usually given special care whilst considering several parameters prior to any surgical intervention and oncological safety is an important factor to keep in mind. Nonetheless, the literature is lacking on data showing the effects of SAL on any residual tumors as in the case of previous positive margins, or the effect of SAL on tumor recurrence and/or metastasis. From what is available, it is only recommended to insure primary tissue transfer (as discussed above). However, all of tumor stage, positive margins, lymphovascular invasions, and the use of vein grafts significantly correlated with worse overall survival and flap complications $[44,45]$, Furthermore, even though all recipient arteries may potentially be in 
immediacy to the primary tumor or its metastatic counterpart, random recipient vessel selection and biopsy has no impact on treatment or prognosis (local cancer recurrence, regional cancer recurrence, or distant metastases) [46]. It is however, recommended to biopsy grossly abnormal recipient vessel that are apparent at the time of flap transfer to determine the pathological status of recipient vessel trimmings [46].

\section{Conclusion}

Suction assisted lipectomy (SAL) is a valuable tool in head and neck contouring after microsurgical free flap reconstruction of oncological defects. The risk of pedicle or perforators injury is minimal, and cosmetic and functional outcomes are favorable. Knowledge of pedicle location is a strong pre-requisite, and the use of blunt tip cannulas is recommended. Prospective studies assessing the outcomes of SAL while controlling the parameters around primary flap transfers are needed to better evaluate the outcomes of SAL, the risk factors and optimal parameters needed, as well as oncological safety measures to be taken.

\section{References}

1. Bui DT, Mehrara BJ, Disa JJ, Cordeiro PG (2004) Use of liposuction for secondary revision of irradiated and nonirradiated free flaps. Ann Plast Surg 52: 541-545.

2. Wooden WA, Shestak KC, Newton ED, Ramasastry SS (1993) Liposuction-assisted revision and recontouring of free microvascular tissue transfers. Aesthetic Plast Surg 17: 103-107.

3. Cigna E, Sassu P, Varkey P, Tan NC, Liu YT (2005) Debulking of free perforator flaps for head and neck reconstruction using an arthroscopic shaver. Ann Plast Surg 55: 441.

4. Cigna E, Minni A, Barbaro M, Attanasio G, Sorvillo V, et al. (2012) An experience on primary thinning and secondary debulking of anterolateral thigh flap in head and neck reconstruction. Eur Rev Med Pharmacol Sci 16: 1095-1101.

5. Hallock GG (1985) Defatting of flaps by means of suction-assisted lipectomy. Plast Reconstr Surg 76: 948-952.

6. Hallock GG (2004) Conventional liposuction-assisted debulking of muscle perforator flaps. Ann Plast Surg 53: 39-43.

7. Huang SH, Wu SH, Chang KP, Wang WH, Lai CH, Sun IF, Lin SD, Lai CS (2009) Contour refinements of free flaps for optimal outcome in oral reconstruction: combination of modified liposuction technique and wplasty in one-stage procedure. Journal of cranio-maxillo-facial surgery: official publication of the European Association for Cranio-MaxilloFacial Surgery 37: 201-205.

8. Teimourian B, Kroll SS (1984) Subcutaneous endoscopy in suction lipectomy. Plast Reconstr Surg 74: 708-711.

9. Hallock GG (1986) Liposuction for debulking free flaps. J Reconstr Microsurg 2: 235-239.

10. Baird W, Nahai F (1989) The use of lipoplasty in contouring and debulking of flaps. Clin Plast Surg 16: 395-399.

11. Grazer FM (1983) Suction-assisted lipectomy, suction lipectomy, lipolysis, and lipexeresis. Plast Reconstr Surg 72: 620-623.

12. Hanasono MM, Skoracki RJ, Silva AK, Yu P (2011) Adipofascial perforator flaps for "aesthetic" head and neck reconstruction. Head Neck 33: 1513-1519.

13. Guelinckx PJ, Sinsel NK (2000) Facial contour restoration in BarraquerSimons syndrome using two free anterolateral thigh flaps. Plast Reconstr Surg 105: 1730-1736.

14. Gasparotti M (1992) Superficial liposuction: a new application of the technique for aged and flaccid skin. Aesthetic Plast Surg 16: 141-153.

15. Field LM (1986) Adjunctive liposurgical debulking and flap dissection in neck reconstruction. J Dermatol Surg Oncol 12: 917-920.
16. Costagliola M, Atiyeh B, Rampillon F, Illouz YG, Dibo S (2013) Aesthetic or functional indications for liposuction. Aesthet Surg J 33: 1212-1213.

17. Teng L, Jin X, Wu G, Zhang Z, Ji Y, et al. (2010) Correction of hemifacial atrophy using free anterolateral thigh adipofascial flap. J Plast Reconstr Aesthet Surg 63: 1110-1116.

18. Teimourian B, Adham MN, Gulin S, Shapiro C (1983) Suction lipectomy--a review of 200 patients over a six-year period and a study of the technique in cadavers. Ann Plast Surg 11: 93-98.

19. Hofer SO, Payne CE (2010) Functional and Aesthetic Outcome Enhancement of Head and Neck Reconstruction through Secondary Procedures. Semin Plast Surg 24: 309-318.

20. Yamanaka K, Ichikawa T, Horiuchi Y (1997) Flap defatting with an ultrasonic surgical aspirator. Plast Reconstr Surg 99: 888-891.

21. Blondeel PN, Derks D, Roche N, Van Landuyt KH, Monstrey SJ (2003) The effect of ultrasound-assisted liposuction and conventional liposuction on the perforator vessels in the lower abdominal wall. $\mathrm{Br} \mathrm{J}$ Plast Surg 56: 266-271.

22. Iverson RE, Lynch DJ; American Society of Plastic Surgeons Committee on Patient Safety (2004) Practice advisory on liposuction. Plast Reconstr Surg 113: 1478-1490.

23. Zhang Q, Qiao Q, Zhou G, Robb GL (2010) Anterolateral thigh adipofascial flap for correction of facial contour deformities and micromastia. J Reconstr Microsurg 26: 341-345.

24. Wolff KD, Kesting M, Loffelbein D, Holzle F (2007) Perforator-based anterolateral thigh adipofascial or dermal fat flaps for facial contour augmentation. Journal of reconstructive microsurgery 23: 497-503.

25. Wang X, Qiao Q, Liu Z, Zhao R, Zhang H, et al. (2005) Free anterolateral thigh adipofascial flap for hemifacial atrophy. Ann Plast Surg 55: 617-622.

26. Tuncali D, Baser NT, Terzioglu A, Aslan G (2007) Romberg's disease associated with Horner's syndrome: contour restoration by a free anterolateral thigh perforator flap and ancillary procedures. Plastic and reconstructive surgery 120 : $67 \mathrm{e}-72 \mathrm{e}$.

27. Jin X, Teng L, Xu J, Lu J, Zhang C, et al. (2010) Anterolateral thigh adipofascial flap for the restoration of facial contour deformities. Microsurgery 30: 368-375.

28. Agostini T, Agostini V (2009) Adipofascial versus fasciocutaneous anterolateral thigh flap in oral cavity reconstruction. Focus on the vascular supply. Journal of plastic, reconstructive \& aesthetic surgery: JPRAS 62: e633-634.

29. Agostini T, Agostini V (2009) Adipofascial anterolateral thigh free flap for hemifacial atrophy. Acta otorhinolaryngologica Italica : organo ufficiale della Societa italiana di otorinolaringologia e chirurgia cervicofacciale 29: 103-107.

30. Papel ID (1992) Facial plastic and reconstructive surgery. Otolaryngol Head Neck Surg 106: 9.

31. Ellsworth WA, Reece GP, Friedman JD (2010) Safety and efficacy of suction-assisted lipectomy on irradiated skin and subcutaneous tissue. Ann Plast Surg 64: 442-445.

32. Agostini T, Russo GL, Zhang YX, Spinelli G, Lazzeri D (2013) Adipofascial anterolateral thigh flap safety: applications and complications. Arch Plast Surg 40: 91-96.

33. Masaki F (2003) Correction of hemifacial atrophy using a free flap placed on the periosteum. Plast Reconstr Surg 111: 818-820.

34. Ibrahim AE, Dibo SA, Hayek SN, Atiyeh BS (2011) Reverse tissue expansion by liposuction deflation for revision of post-surgical thigh scars. International wound journal 8: 622-631.

35. Guelinckx PJ, Boeckx WD, Fossion E, Gruwez JA (1984) Scanning electron microscopy of irradiated recipient blood vessels in head and neck free flaps. Plast Reconstr Surg 74: 217-226.

36. Bernstein H (1964) Treatment of Keloids by Steroids with Biochemical Tests for Diagnosis and Prognosis. Angiology 15: 253-260.

37. Donal PJ (2010) Difficult Case in Head and Neck Cancer surgery. Thieme 
Citation: Ibrahim AE, Janom H, Raad M (2015) Liposuction Contouring After Head and Neck Free Flap Reconstruction. Anaplastology 4: 145.

Page 5 of 5

38. Bozikov K, Arnez ZM (2006) Factors predicting free flap complications in head and neck reconstruction. J Plast Reconstr Aesthet Surg 59: 737-742.

39. Eckardt A, Fokas K (2003) Microsurgical reconstruction in the head and neck region: an 18-year experience with 500 consecutive cases. Journal of cranio-maxillo-facial surgery: official publication of the European Association for Cranio-Maxillo-Facial Surgery 31: 197-201.

40. Okazaki M (2012) Functional and Aesthetic Reconstruction of the Defects Following the Hemiglossectomy in Patients with Oropharyngeal Cancer.

41. Clark JR, McCluskey SA, Hall F, Lipa J, Neligan P, et al. (2007) Predictors of morbidity following free flap reconstruction for cancer of the head and neck. Head Neck 29: 1090-1101.

42. Newman MI, Hanasono MM, Disa JJ, Cordeiro PG, Mehrara BJ (2004) Scalp reconstruction: a 15-year experience. Ann Plast Surg 52: 501-506.
43. Van Driel AA, Mureau MA, Goldstein DP, Gilbert RW, Irish JC, et al. (2010) Aesthetic and oncologic outcome after microsurgical reconstruction of complex scalp and forehead defects after malignant tumor resection: an algorithm for treatment. Plastic and reconstructive surgery 126: 460-470.

44. Chang EI, Yu P, Skoracki RJ, Liu J, Hanasono MM (2015) Comprehensive Analysis of Functional Outcomes and Survival after Microvascular Reconstruction of Glossectomy Defects. Annals of surgical oncology.

45. Miller MJ, Schusterman MA, Reece GP, Kroll SS (1993) Interposition vein grafting in head and neck reconstructive microsurgery. J Reconstr Microsurg 9: 245-251.

46. DeConde AS, Thompson CF, Zaghi S, Vira D, Blackwell KE, et al. (2014) Oncologic validity of recipient vessel preservation in microvascular freeflap reconstruction. ORL J Otorhinolaryngol Relat Spec 76: 193-198. 